\title{
Physicochemical Characteristics of Natural Fruit and Vegetable Fermentation Broth and Monitoring of Its Fermentation Process
}

\author{
Miao Li ${ }^{1,2, ~ *, ~ H a o ~ S o n g ~}{ }^{1,2}$ \\ ${ }^{1}$ Beijing Industrial Technology Research Institute, Beijing, China \\ ${ }^{2}$ Beijing Food Industry Research Institute, Beijing, China \\ Email address: \\ limiao0103@126.com (Miao Li), lyfd2002@163.com (Hao Song) \\ ${ }^{*}$ Corresponding author
}

\section{To cite this article:}

Miao Li, Hao Song. Physicochemical Characteristics of Natural Fruit and Vegetable Fermentation Broth and Monitoring of Its Fermentation Process. Science Discovery. Vol. 7, No. 4, 2019, pp. 200-204. doi: 10.11648/j.sd.20190704.14

Received: July 4, 2019; Accepted: July 25, 2019; Published: July 29, 2019

\begin{abstract}
The natural fermentation broth of fruits and vegetables is the fermentation broth obtained from the natural fermentation process of fruits and vegetables. The raw fermentation broth is produced by anaerobic fermentation of fruits and vegetables, so as to form a fermentation beverage with complex components, which can promote digestion and absorption, alleviate constipation and beautify the face. Fruit fermentation can be used not only as a drink, but also as a substitute for highly active dry yeast. It can be added into bread to make natural yeast bread with good flavor and long shelf life and other baking products. It has broad market application prospects. In this paper, physical and chemical indexes (soluble solids content, alcohol content, total acid content) and microbial indexes of natural fermentation products on sale and self-made fermentation broth of fruits and vegetables were determined, and the morphology, size and color of yeast colony in fermentation broth were observed. In order to find the suitable fermentation time, the fermentation processes of two kinds of compound fermentation broth of fruits were monitored continuously and dynamically. The results showed that the contents of soluble solids, alcoholicity, total acid content and yeast count were significantly different among different natural fermentation liquors of fruits and vegetables due to different raw materials, formulations and fermentation time. The content of soluble solids in compound natural fermentation broth 1 (orange + apple + lemon) was reduced from $13.1 \%$ to $4.0 \%$ and the $\mathrm{pH}$ was decreased from 4.2 to 3.1 , then slowly increased to 3.3 during the 40 -day fermentation at $28^{\circ} \mathrm{C}$, while the content of soluble solids in compound natural fermentation broth 2 (apple + pear + lemon) decreased slowly from $12.4 \%$ to $8.5 \%$ and the $\mathrm{pH}$ decreased from 4.4 to 2.9 at the same fermentation temperature and time.
\end{abstract}

Keywords: Fruit and Vegetable Fermentation Broth, Natural Fermentation, Soluble Solid Content

\section{天然果蔬酵液的理化特性及其发酵过程的监测}

\author{
李妙 ${ }^{1,2 *}$, 宋昊 ${ }^{1,2}$ \\ ${ }^{1}$ 北京一轻研究院, 北京, 中国 \\ 2 北京市食品工业研究所, 北京, 中国
}

邮箱

limiao0103@126.com（李妙）, 1yfd2002@163.com(宋吴)

摘要: 果蔬天然酵液为果蔬经过天然发酵过程得到的发酵液, 将水果、蔬菜等食用材料, 经过厌氧发酵过程酿制产生食物 发酵原液, 以形成一种具有复合成分, 能够促进消化吸收、缓解便秘、美容养颜的酵液饮品。水果酵液除作为饮料直接饮 用外，亦可代替高活性干酵母，添加入面包中，制成风味好、货架期长的天然酵母面包以及其他烘焙产品，具有广阔的市 
场应用前景。本文对市售果蔬天然酵液产品和自制酵液进行理化指标（可溶性固形物含量、酒精度、总酸含量）及微生物 指标的测定，观察酵液中酵母菌的菌落形态、大小、颜色等特征，并对两款复合水果酵液的发酵过程进行持续的动态监测， 以寻找合适的发酵时间。结果表明，不同的果蔬天然酵液之间由于原料、配方、发酵时长不同，可溶性固形物含量、酒精 度、总酸含量、酵母菌总数等指标差异较大。自制的复合天然酵液 1 （橙子+苹果 + 柠檬）在 $28^{\circ} \mathrm{C}$ 恒温条件下，在 40 天的发 酵过程中, 可溶性固形物含量由初始的 $13.1 \%$ 降至 $4.0 \%, \mathrm{pH}$ 由 4.2 降至 3.1 , 又缓慢上升至 3.3 ; 复合天然酵液2（苹果+梨+柠 檬）在相同的发酵温度和发酵时长中，可溶性固形物含量由初始的 $12.4 \%$ 缓慢、持续降至 $8.5 \%, \mathrm{pH}$ 由 4.4 降至 2.9 。

关键词：果蔬酵液，天然发酵，可溶性固形物含量

\section{1. 引言}

果蔬天然酵液是将一种或数种水果、蔬菜为原料，经 清洗、切片后密封发酵制成的植物发酵制品。果蔬按一定 配比添加白砂糖、蜂蜜等, 在厌氧条件下经数种酵母、醋 酸菌、乳酸菌等微生物发酵, 经数十日发酵而成。果蔬天 然酵液具有预防或降低一些慢性疾病发生概率的作用, 植 物原料可以提供许多营养物质和 “植物类功能性化学成 分”, 微生物发酵生成各种生理活性物质, 如多糖、氨基 酸、多肽、维生素、多酚类、有机酸、醇类、酯类、酶类、 黄酮类物质等, 以及GABA（ $\gamma$-aminobutyric acid, $\gamma$-氨 基丁酸)、SOD (Superoxide Dismutase, 超氧化物歧化酶)、 过氧化氢酶等抗氧化物质 [1-4], 同时提供多种益生菌, 营 养丰富, 有益于肠道健康, 解酒护肝, 具有抗氧化作用, 增强机体的免疫力 $[2,5-11]$ 。

果蔬天然酵液中的益生菌, 能够改善和平衡人体肠道菌 群, 抑制肠道中病原菌的繁殖, 促进肠道菌群正常化, 诱导 产生干扰素, 活化免疫细胞, 提高机体的免疫力。酵液中的 益生元也可以调节肠道菌群的活性, 抑制肠道腐败[12]。酵 液中的益生菌和益生元除了在人体肠道中发挥保健作用外, 亦可作为高活性干酵母的替代物, 添加至面包等烘焙产品中, 酵液中的多糖、氨基酸、多肽、有机酸、醇类、酯类等物质 可以改善面包的风味, 益生菌、益生元可以抑制杂菌的生长、 繁殖, 起到延长面包保质期、防止面包老化的作用。因此, 果蔬天然酵液不仅可以作为饮料, 直接饮用 [13], 也可以代 替活性酵母，用于面包等烘焙产品的制作。

岛本觉也, 日本磐亚株式会社的微生物专家, 从柴田 欣司氏应用微生物生产农肥中, 分离出了二十多种有益菌, 并将其按一定配比混合, 制成了酵素菌原菌[14]。二十世 纪末被多个国家引进推广, 广泛应用于食品加工业、养殖 业、种植业等领域。

日本的酵液制作工艺较为成熟，但风味尚存在提升空 间。国内对果蔬酵液的研发处在发展阶段 $[15,16]$, 存在巨 大的发展空间与市场潜力。

\section{2. 材料和方法}

\section{1. 材料与试剂}

橙子、苹果、梨、柠檬: 京东生鲜。

葡萄干：乌鲁木齐市汇锦果源果品有限公司。 怡宝饮用纯净水：华润怡宝饮料（中国）有限公司。
白砂糖：上海市糖业烟酒（集团）有限公司。

酵冠水果液（提子桂圆味）: 神木市长青健康农产业 发展有限公司。

酵冠水果液 (果蔬味) : 神木市长青健康农产业发展 有限公司。

平板计数琼脂（Plate Count Agar, PCA）：北京陆桥 技术股份有限公司。

酵母浸粉: 北京奥博星生物技术有限责任公司。

蛋白炼: 国药集团化学试剂有限公司。

葡萄糖: 国药集团化学试剂有限公司。

琼脂粉：国药集团化学试剂有限公司。

氢氧化钠: 国药集团化学试剂有限公司。

氯化钠: 国药集团化学试剂有限公司。

消毒酒精: 杭州欧拓普生物技术有限公司。

精密 $\mathrm{pH}$ 试纸: 上海三爱思试剂有限公司。

生理盐水: $0.9 \% \mathrm{NaCl}$ 。

擦镜纸: 杭州特种纸业有限公司。

\section{2. 仪器与设备}

生化培养箱: 上海一恒科学仪器有限公司。 电热恒温水浴锅: 上海一恒科学仪器有限公司。 高压灭菌锅: 重庆雅马拓科技有限公司。 洁净工作台：苏净集团苏州安泰空气技术有限公司。 电子天平: Sartorius公司。

微量移液器及枪头: Eppendorf公司。

计数器：深圳市与非投资发展有限公司。

发酵桶: 北京天伦华谊科技发展有限公司。

PAL-3便携式数显糖度计：日本爱拓Atago公司。

培养血: 直径 $90 \mathrm{~mm}$ 。

酒精计。

全玻璃蒸馏器。

雉形瓶、试管、移液管、均质杯。

\section{3. 方法与步骤}

\subsection{1. 配方}

表1 复合酵液1配方。

\begin{tabular}{ll}
\hline 配料 & 重量/体积 \\
\hline 橙子(去皮) & $950 \mathrm{~g}$ \\
苹果 & $200 \mathrm{~g}$ \\
柠檬 & $50 \mathrm{~g}$ \\
白砂糖 & $750 \mathrm{~g}$ \\
纯净水 & $5.5 \mathrm{~L}$ \\
\hline
\end{tabular}


表2 复合酵液2配方。

\begin{tabular}{ll}
\hline 配料 & 重量/体积 \\
\hline 苹果 & $600 \mathrm{~g}$ \\
梨 & $550 \mathrm{~g}$ \\
柠檬 & $50 \mathrm{~g}$ \\
白砂糖 & $750 \mathrm{~g}$ \\
纯净水 & $5.5 \mathrm{~L}$ \\
\hline
\end{tabular}

表3 葡萄干酵液配方。

\begin{tabular}{ll}
\hline 配料 & 重量/体积 \\
\hline 葡萄干 & $400 \mathrm{~g}$ \\
白砂糖 & $200 \mathrm{~g}$ \\
水 & $0.8 \mathrm{~L}$ \\
\hline
\end{tabular}

表4 YPD(Yeast Extract Peptone Dextrose Medium)培养基。

\begin{tabular}{ll}
\hline 配料 & 重量/体积 \\
\hline 酵母浸粉 & $1 \%$ \\
蛋白胨 & $2 \%$ \\
葡萄糖 & $2 \%$ \\
琼脂 & $2 \%$ \\
去离子水 & $1 \mathrm{~L}$ \\
\hline
\end{tabular}

YPD培养基 $115^{\circ} \mathrm{C}$ 高压蒸汽灭菌 $30 \mathrm{~min}$ 后备用。

\subsection{2. 操作步骤}

（1）水果天然酵液的制备:

(1)选果及清洗: 依各原料组成选取新鲜、成熟的水果 (病虫害果、霉烂果、未成熟果不能选用), 分别用自来 水及去离子水清洗干净。操作全程需佩戴乳胶手套、口罩, 避免口腔及皮肤表面的微生物混入水果酵液中, 影响其自 然发酵或产生霉变。

(2)器具消毒: 将果盘、刀、发酵桶用洗洁精、自来水 及去离子水清洗干净, 并用 $75 \%$ 乙醇喷洗、消毒。

(3)切片: 将清洗干净的各类水果去皮去核, 放入已消 毒的果盘中, 切成薄厚均匀、大小适宜的薄片。

(4)将水果切片、白砂糖、纯净水按照配方（表1 表3） 比例放入发酵桶中, 经摚拌均匀将盖子盖紧密封存放 (发 酵桶内的空气可自行排出, 发酵桶外的空气不可进入桶 内）。

(5)将发酵桶置于 $28^{\circ} \mathrm{C}$ 恒温箱中进行自然发酵。

(2) 可溶性固形物含量测定:

(1)将去离子水滴至折光仪镜面中央, 充满凹陷处, 按 下折光仪上调零按钮 (ZERO)，使得读数为 0 , 然后用擦 镜纸擦净镜面和滴液仓。

(2)将待测的果蔬天然酵液用滴管滴至折光仪镜面中 央, 使试液均匀无气泡, 充满滴液仓, 按下折光仪上START 按钮, 记录Brix读数及温度。

(3) 乙醇浓度的测定:

(1)用干燥、洁净的 $200 \mathrm{~mL}$ 容量瓶准确量取 $200 \mathrm{~mL}$ 样 品于 $500 \mathrm{~mL}$ 蒸馏瓶中, 用 $50 \mathrm{~mL}$ 去离子水分三次冲洗容量 瓶, 倒入蒸馏瓶中。

(2)向蒸馏瓶中加入沸石, 连接蛇形冷凝管, 开启冷却 水, 缓慢加热蒸馏, 用取样用的原容量瓶收集馏出液。

(3)将馏出液倒入干燥、洁净的 $200 \mathrm{~mL}$ 量筒中, 放入干 燥、洁净的酒精计, 轻按一下, 同时放入干燥、洁净的温
度计, 平衡 $5 \mathrm{~min}$ 左右, 水平观测, 读取弯月面相切处的 刻度值, 并记录温度。

(4) 根据温度和测得的酒精计读数, 查询 GB 5009.225-2016附录B，换算成 $20^{\circ} \mathrm{C}$ 时试液的酒精度。

(4) 总酸的测定 (以柠檬酸计) :

(1)称取 $1 \mathrm{~g}$ 酚酞, 溶解于 $60 \mathrm{~mL} 95 \%$ 乙醇中, 用双蒸水 稀释至 $100 \mathrm{~mL}$ 。

(2)将果蔬天然酵液过滤, 收集滤液。

(3)称取 $50 \mathrm{~g}$ 试液, 置于 $250 \mathrm{~mL}$ 锥形瓶中, 加入 $50 \mathrm{~mL}$ 双蒸水, $0.2 \mathrm{~mL} 1 \%$ 酚酞指示剂, 用 $0.01 \mathrm{~mol} / \mathrm{L} \mathrm{NaOH}$ 标准 滴定溶液滴定至微红色, 且30 $\mathrm{s}$ 内不褪色, 记录消耗的 $\mathrm{NaOH}$ 溶液的体积。

(4)空白实验: 用双蒸水代替试液, $0.01 \mathrm{~mol} / \mathrm{L} \mathrm{NaOH}$ 标准滴定溶液滴定至微红色, 且30 s内不裉色, 记录消耗 的 $\mathrm{NaOH}$ 溶液的体积。

(5) 酵母菌计数:

(1)用无菌移液管吸取 $25 \mathrm{~mL}$ 样品至盛有 $225 \mathrm{~mL}$ 无菌 生理盐水的均质杯中, 充分振荡混匀, 制成 $1: 10$ 的样品匀 液。

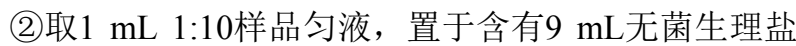
水的试管中, 混匀, 制成 $1: 100$ 的样品匀液。

(3)制备一系列10倍递增稀释样品匀液。

(4)选择 3 个适宜稀释度的样品匀液, 分别取 $1 \mathrm{~mL}$ 置于 2 个无菌培养血内, 倒入 $25 \mathrm{~mL} 46^{\circ} \mathrm{C}$ 的 $\mathrm{PCA}$ 培养基, 转动培 养血混合均匀。

(5)分别取 $1 \mathrm{~mL}$ 无菌生理盐水加入培养血中, 倒入PCA 培养基，作为阴性对照。

(6)待培养基完全凝固后, 置于 $28^{\circ} \mathrm{C}$ 恒温培养箱中培养 $48 \sim 72 \mathrm{~h}$ 。

(7)选取菌落数在10 150 CFU（colony-forming units） 的平板, 进行计数, 计算同一稀释度的两个平板的平均值。

\section{3. 结果与分析}

\section{1. 天然果蔬酵液中理化指标的测定}

选取商品化的酵冠水果液（提子桂圆味）、酵冠水果 液（果蔬味），自制的葡萄干酵液1、葡萄干酵液2（以下 分别简写为酵冠 1 、酵冠 2 、葡萄干 1 、葡萄干2），进行理 化指标测定, 包括可溶性固形物含量、酒精度、总酸、酵 母菌总数四项。

表5 四款果蔬天然酵液理化指标测定结果。

\begin{tabular}{lllll}
\hline 果蔬醉液 & 酵冠1 & 酵冠2 & 葡萄干1 & 葡萄干2 \\
\hline 可溶性固形物 $(\%)$ & 17.5 & 6.5 & 30.5 & 17.5 \\
酒精度 $(\% \mathrm{vol})$ & 10.3 & 6.5 & 4.7 & 8.9 \\
总酸 $(\mathrm{g} / 100 \mathrm{~g})$ & 4.58 & 5.20 & 5.75 & 5.01 \\
酵母菌总数 $\left(\times 10^{6} \mathrm{CFU} / \mathrm{g}\right)$ & 4.0 & 8.0 & 6.5 & 1.6 \\
\hline
\end{tabular}

如表5所示, 市场上所售的酵冠品牌的两款果蔬酵液 (酵冠 1 、酵冠 2 ) 中, 可溶性固形物差异较大, 分别为 $17.5 \%$ 、 $6.5 \%$ 。酒精度分别为 $10.3 \% \mathrm{vol}$ 和 $6.5 \% \mathrm{vol}$ 。总酸含量较为 接近, 分别是 $4.58 \mathrm{~g} / 100 \mathrm{~g} 、 5.20 \mathrm{~g} / 100 \mathrm{~g}$ 。酵母菌总数为 $4.0 \times 10^{6} \mathrm{CFU} / \mathrm{g} 、 8.0 \times 10^{6} \mathrm{CFU} / \mathrm{g}$ 。 
自制的两个葡萄干酵液样品（配方见表 3 ）中，样品 1 （葡萄干1）的可溶性固形物含量较高, 达到了 $30.5 \%$, 推 测发酵尚不完全, 样品2（葡萄干2）中该指标为 $17.5 \%$ 。 二者的酒精度分别是 $4.7 \% \mathrm{vol}$ 和 $8.9 \% \mathrm{vol}$, 也印证了样品 1 (葡萄干1) 中发酵程度较低的猜想。总酸含量较接近, 分别是 $5.75 \mathrm{~g} / 100 \mathrm{~g}$ 和 $5.01 \mathrm{~g} / 100 \mathrm{~g}$ 。二者的酵母菌总数为 $6.5 \times 10^{6} \mathrm{CFU} / \mathrm{g} 、 1.6 \times 10^{6} \mathrm{CFU} / \mathrm{g}$ 。

\section{2. 天然果蔬酵液中微生物的菌落形态}

将六款天然酵液在YPD固体培养基（配方见表4）上 进行划线培养, 使得单个微生物细胞分散开来, 在固体平 面培养基上生长、繁殖形成肉眼可见的群体, $28^{\circ} \mathrm{C}$ 恒温培 养24 h 后观察其菌落形态。如图1中所示，六款酵液中的 微生物菌落大而厚, 呈圆形, 不透明, 大小、形态一致, 质地均匀、湿润, 表面较光滑, 有油脂光泽, 易挑起, 乳 白色, 有一定酒香味, 初步鉴定均为酵母菌, 未出现细菌 和霉菌的菌落特征。
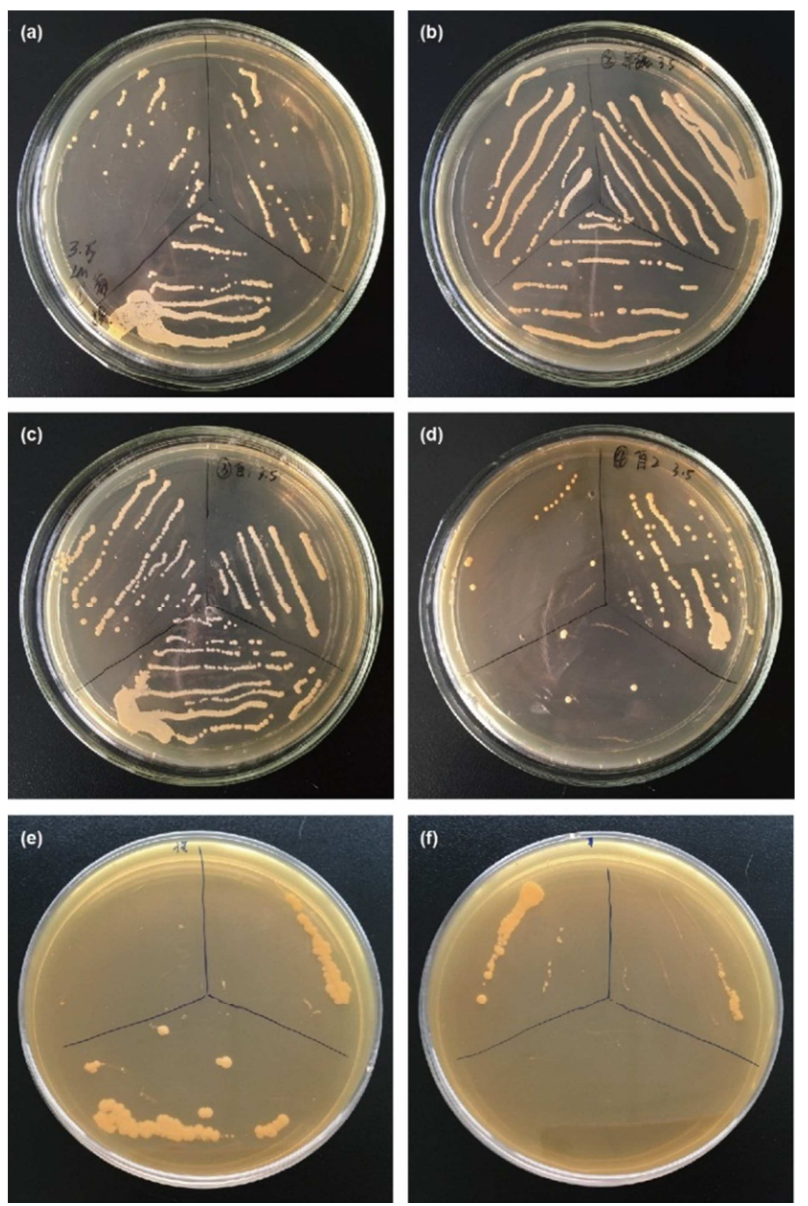

图1 果蔬天然酵液中微生物的菌落特征。（a）酵冠水果液（提子桂圆 味）；（b）酵冠水果液（果蔬味）；（c）葡萄干酵液 1 ；（d）葡萄干 酵液 $2 ; （ \mathrm{e})$ 复合酵液 $1 ; （ \mathrm{f})$ 复合酵液 2 。

\section{3. 两款复合水果酵液发酵终点的确定}

自制的两款复合水果酵液（配方见表1、表2），在恒 温 $28^{\circ} \mathrm{C}$ 条件下放置 40 天, 每天测定其 $\mathrm{pH}$ 和可溶性固形物 含量 (Brix), 结果如图2和图3所示。
第 0 天时，复合酵液 1 的初始可溶性固形物含量为 $13.1 \%$, 发酵的前 15 天可溶性固形物含量下降较快, 呈“S” 形曲线; 第 16 天时, 其含量为 $4.4 \%$, 其后呈缓慢降低趋势; 至第 40 天时, 可溶性固形物含量为 $4.0 \%$ 。复合酵液 1 的初 始 $\mathrm{pH}$ 为 4.2 , 发酵的前 14 天, $\mathrm{pH}$ 一直呈下降趋势, 在第 14 天达到 3.1 后, 基本保持不变, 与可溶性固形物含量的变 化情况基本吻合; 自第27天起, 有缓慢上升趋势, 在第 40 天 $\mathrm{pH}$ 值达到3.3（图2）。

如图3中所示, 复合酵液 2 的初始可溶性固形物含量为 $12.4 \%$, 其后呈阶梯状下降趋势, 在发酵的前 8 天缓慢降低, 其含量达到 $11.9 \%$; 第9天至第 19 天, 可溶性固形物含量下 降较快, 达到 $9.7 \%$; 第 20 天至 27 天, 基本维持不变; 第 28 天至 40 天, 可溶性固形物含量下降至 $8.5 \%$ 。复合酵液 2 的 初始 $\mathrm{pH}$ 值为 4.4 , 发酵的前 7 天有明显降低, 第7天时 $\mathrm{pH}$ 为 3.1 ; 第 8 天至第 40 天, $\mathrm{pH}$ 有缓慢下降趋势, 第 40 天为 2.9 。

根据可溶性固形物含量随时间的变化情况来看, 两款 复合水果酵液的发酵情况不同: 复合酵液 1 的主要成分为 橙子, 所需的发酵时间较短, 且最终的可溶性固形物含量 较低, 第 15 天可作为其发酵终点; 复合酵液 2 的主要成分 为苹果和梨, 在 40 天中持续缓慢发酵, 可溶性固形物含量 维持较高的状态。这些动态变化结果，对于之后开发复合 水果酵液饮料和天然酵母面包具有参考价值和指导意义。

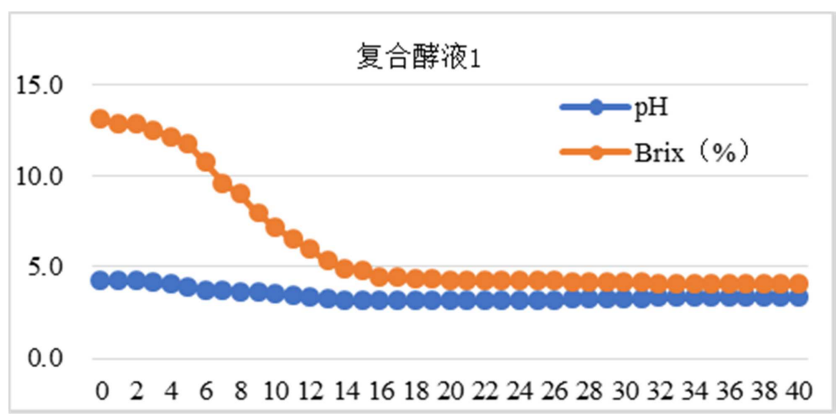

图2 复合水果酵液 1 的 $\mathrm{pH}$ 、Brix随时间的变化情况。

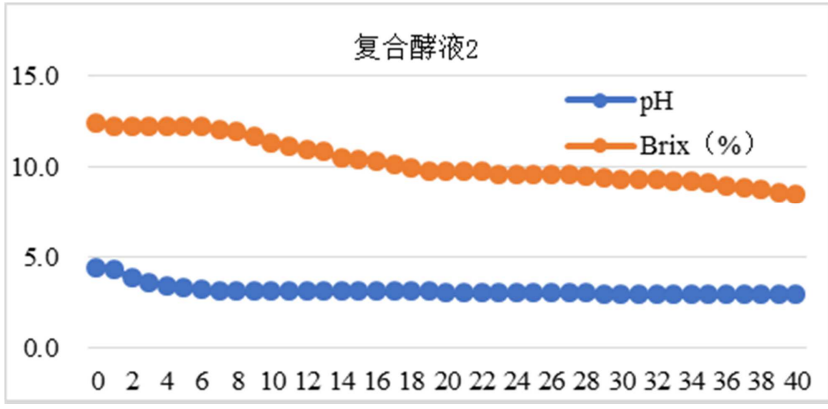

图3 复合水果酵液 2 的 $\mathrm{pH}$ 、Brix随时间的变化情况。

\section{4. 结论}

不同的果蔬天然酵液具有不同的理化指标 (可溶性固 形物含量、酒精度、总酸含量) 和酵母菌种类、数量, 其 风味和发酵情况也不尽相同。本文通过对市售的果蔬天然 酵液产品、自制单一酵液（葡萄干酵液）、自制复合酵液 (橙子+苹果+柠檬、苹果+梨+柠檬) 的理化指标和酵母 菌菌落形态进行观测, 并对天然酵液发酵的动态过程进行 
监测, 确定复合酵液 1 可在恒温 $28^{\circ} \mathrm{C}$ 条件下进行为期 15 天 的发酵, 复合酵液 2 可进行长时间（40天以上）的发酵, 其后可以针对这两款天然酵液做进一步的研发, 如制成酵 液饮料、作为高活性干酵母的替代物加入面包等烘焙产品 中等。

\section{致谢}

本文为北京一轻产业扶植培育基金项目《活性益生菌 的发酵研究及其产品开发》的阶段性成果之一。

\section{参考文献}

[1] 胡学智.酵素㙏[N].中国食品报, 2016。

[2] 董银卯, 何聪芬, 王领, 等. 火龙果酵素生物活性的初步研 究[J].食品科技，2009，34(03)：192-196。

[3] 苏龙, 庄明川, 陆孔泳, 等. 芒果木瓜天然酵素抗氧化性能 分析[J].湖北农业科学, 2017, 56(07): 1312-1314。

[4] 郭红莲, 邢紫娟, 余巧银, 乔博金鈄. 天然枸杞酵素发酵的代 谢产物分析[J].食品研究与开发，2018，39(05)：48-55。

[5] 赵金凤, 宿秀芹, 曲佳乐, 等.敖东酵素对脾淋巴细胞增殖 作用的影响 $[\mathrm{J}]$.食品与发酵科技，2014，50(3)：50-52。

[6] 曲佳乐, 赵金风, 皮子凤, 等.植物酵素解酒护肝保健功能 研究 $[J]$.食品科技, 2013，38(09)：51-55。
[7] 蒋增良, 毛建卫, 黄俊, 等. 葡萄酵素在天然发酵过程中体 外抗氧化性能的变化 [J].中国食品学报, 2014, 14(10): 29-34。

[8] 冯莉, 张鹤金金, 何国库, 等.水果酵素对小鼠酒精性肝损伤 保护作用的研究[J].中国酿造, 2017，36(9)：112-115。

[9] 格瑞宏, 储瑞蔼, 李井泉, 王慧. 桂圆酵素制备及其抗氧化 性研究[J].食品科技，2015，40(08)：262-267。

[10] 郭艳萍, 赵金安. 大麦天然酵素抗氧化性能的初步探究 [J]. 山西化工，2014，34(04): 4-6。

[11] 贾丽丽, 冀利, 孙曙光, 王亚妮. 冬束酵素发酵过程中生物 学特性和抗氧化活性研究 [J]. 食品与发酵科技, 2014, 50(04): 30-33。

[12] 毛建卫, 吴元锋, 方晟. 微生物酵素研究进展 [J]. 发酵科技通 讯, 2010，39(3): 42-44。

[13] 颜晓庆, 崔红燕, 陈宏运, 等. 植物发酵液在功能性运动饮 料中的应用 [J].食品与发酵工业，2016，42(1)：277-280。

[14] 岛本邦彦.岛本生物农业应用法[M].郑重, 潍坊: 酵素菌素 世界社中国山东总部，1996：5-15。

[15] 王楠, 王锦, 许珺.酵素技术及在我国的应用: 中国科学技 术协会学会学术部.第十六届中国科协年会一分 5 生态环 境保护与绿色发展研讨会论文集[C].中国云南昆明: 中国科 学技术协会、云南省人民政府, 2014：156-159。

[16] 陈倩, 刘善江, 李亚星.我国酵素菌技术概况及应用现状 [J]. 安徽农业科学，2012，40(23)：11612-11615。 